\title{
Impacted mandibular third molars: the efficacy of prophylactic antibiotics and chlorhexidine mouthwash in preventing postoperative infections
}

SADJ June 2017, Vol 72 no 5 p213 - p218

P Gopee, ${ }^{1}$ E Rikhotso ${ }^{2}$

\section{ABSTRACT}

Introduction: There are dissenting opinions regarding the efficacy of prophylactic antimicrobial agents in third molar surgery.

Objective: To determine: 1) The efficacy of a prophylactic antibiotic regimen compared with a chlorhexidine mouthwash in reducing postoperative infections in mandibular third molar surgery. 2) The pattern of presentation and the indications for extraction of mandibular third molars.

Design: 100 surgical patients were randomly assigned to two groups (group 1: $15 \mathrm{ml}$ of chlorhexidine rinse for one minute preoperatively. group $2: 2 \mathrm{~g}$ amoxicillin orally one hour preoperatively).

Methods: Postoperative complications and surgical site infections were assessed seven days postoperatively. The patients' age, gender, type of impaction and indications for extraction were also recorded. Data was analyzed using the statistical package STATA 13.1.

Results: The infection rate was $8 \%$ and $6 \%$ for groups 1 and 2 respectively. No statistically significant difference in surgical wound infection was found between the two groups.

Mesioangular impaction was the most common type of impaction, and pericoronitis was the most prevalent indication for extraction.

Conclusion: Amoxicillin and chlorhexidine prophylaxis are equally effective in reducing postoperative infections in third molar surgery. Hence, antibiotic prophylaxis is not indicated for routine administration in non-immunocompromised patients for such procedures.

1. Pooshan Gopee: BDS, MSc Dent (Wits). Dentist, Ermelo Provincial Hospital, Ermelo.

2. Ephraim Rikhotso: BDS, MDent (MFOS), FCMFOS. Head of Department, Maxillofacial and Oral Surgery, University of the Witwatersrand.

Corresponding author

Pooshan Gopee:

Ermelo Provincial Hospital, Private Bag, X9005, Ermelo, 2350.

Cell: 076470 3139. Email: tavishg@yahoo.com

\section{ACRONYMS}

ADA*: American Dental Association

AHA: American Heart Association

AAOS: American Academy of Orthopaedic Surgeons

BSAC*: British Society for Antimicrobial Chemotherapy

WHO*: World Health Organisation

\section{INTRODUCTION}

Removal of impacted teeth is a very common surgical procedure performed by Oral and Maxillofacial Surgeons as well as general dentists in the South African Public Health sector. ${ }^{1}$ Owing to the complexity of this surgical procedure, extensive skills and training are required in order to reduce the risk of complications that may arise during or after surgery. ${ }^{1}$ In addition, adequate knowledge regarding the diagnostic and treatment modalities is essential in order to achieve optimum results for the patients.

Complications associated with third molar surgery can be classified as either infection or non-infection related. ${ }^{2}$ Non-infection related complications usually feature pain, swelling and erythema due to the normal inflammatory process following surgical trauma, while complications that occur with infection include alveolar osteitis, osteomyelitis, lymphadenopathy (localized/generalized) and fascial space involvement. ${ }^{2}$

Traditionally, antibiotic therapy has been used to reduce bacterial contamination and subsequent infection at the surgical site. A plethora of studies exists in the literature regarding the efficacy of antibiotic therapy in reducing postoperative infection in third molar surgery. ${ }^{3,4}$ Other studies have however demonstrated that prophylactic antibiotics may not be of any value in reducing the incidence of infection. ${ }^{5-9}$

The absence of broad-based consensus on the efficacy of antibiotic prophylaxis in third molar surgery has resulted in the proliferation of haphazard and unsystematic prescribing of antibiotics among dental practitioners; despite the low risk of complication associated with this surgery. 
Antimicrobial mouthwash agents have for long been used for prophylaxis or treatment of oral infections in dentistry. However, their usefulness in reducing postoperative infections in third molar surgery has often been overlooked, probably due to the limited number of studies which have been carried out to assess their efficacy. ${ }^{10-12}$

Against this background, the current study was designed to evaluate the efficacy of prophylactic antibiotics and a chlorhexidine mouthwash in preventing postoperative infections following removal of mandibular third molar teeth. Furthermore, an attempt was made to generate a pool of data not only in the demographics and distribution patterns of patients presenting with impacted third molar teeth but also in treatment outcomes after extraction. Such data are essential in establishing evidence based clinical guidelines that can further assist in the development of sound treatment protocols.

\section{MATERIALS AND METHODS}

\section{Study Design}

A prospective randomized controlled trial was conducted on patients who presented to the Oral and Dental Department of Ermelo Provincial and Rob Ferreira hospitals for third molar extractions under local anaesthesia between October 2014 and October 2015. The study was approved by the Human Research Ethics Committee (HREC) of the University of the Witwatersrand (Certificate No. M140435). Medically competent patients, with ages ranging from 18-50 years were included in the study after each had signed an Informed Consent declaration. Exclusion criteria were defined as patients having comorbidities or third molar teeth with incomplete root formation. The surgical procedures were timed and those with an operating time above one hour were excluded from the study sample.

\section{Population}

The study sample was derived from patients who reported for mandibular third molar extractions at one of the two hospitals. One hundred and ten patients requiring third molar surgical extractions were recorded for the study. Of that total, 100 patients met the inclusion criteria. Seven patients did not attend the recall appointment and three patients had undergone an operating time of more than one hour.

To test the effectiveness of the prophylactic regimen in the study, patients were randomly assigned to two groups (using two sealed envelopes). In the first group (control group), patients were instructed to rinse with $15 \mathrm{ml}$ of $0.2 \%$ chlorhexidine (with alcohol) mouthrinse for one minute prior to the surgery. In the second group (test group), $2 \mathrm{~g}$ amoxicillin was administered orally one hour before the surgery.

The Control group consisted of 50 patients; 22 males and 28 females.

The Test group consisted of 50 patients; 26 males and 24 females.

All the operations were of a surgical nature and were performed under local anaesthesia (2\% lignocaine with $1: 80000$ adrenaline) by a single operator with six years of experience in using a standardized procedure.

The surgery was carried out based on the university's protocol on third molar extraction. Envelope or triangular mucoperiosteal flap elevation with ostectomy and/ or odontectomy was achieved using a surgical scalpel blade no. 15. Ostectomy was performed using a crosscut tapered fissure bur mounted on a straight handpiece. The tooth was sectioned appropriately where necessary depending on the surgeon's judgement whilst trying to achieve minimal surgical invasion.

Following the removal of the tooth, the surgical site was debrided and irrigated with sterile water. Primary closure of the flap was achieved using resorbable chromic catgut sutures, 3/0.

The time of surgery was recorded as the time span between the first incision and the last suture placed.

\begin{tabular}{|c|c|c|}
\hline Variable & Frequency & $\%$ \\
\hline \multicolumn{3}{|l|}{ Sex } \\
\hline Female & 52 & 52.00 \\
\hline Male & 48 & 48.00 \\
\hline Age (mean, std) & \multicolumn{2}{|c|}{$27.75(5.79)$} \\
\hline \multicolumn{3}{|l|}{ Type of Impaction } \\
\hline Vertical & 37 & 37.00 \\
\hline Horizontal & 14 & 14.00 \\
\hline Mesioangular & 46 & 46.00 \\
\hline Distoangular & 3 & 3.00 \\
\hline \multicolumn{3}{|l|}{ Indication for Extraction } \\
\hline Pericoronitis & 39 & 39.00 \\
\hline Abscess & 3 & 3.00 \\
\hline Caries & 34 & 34.00 \\
\hline Periodontal disease & 10 & 10.00 \\
\hline Root resorption & 2 & 2.00 \\
\hline Atypical facial pain & 6 & 6.00 \\
\hline Oral pathology & 1 & 1.00 \\
\hline Prophylactic removal & 4 & 4.00 \\
\hline Tooth in line of fracture & 1 & 1.00 \\
\hline
\end{tabular}

Time taken in minutes (mean, std) 22.14 (9.69)

\section{Medication}

\section{Antibiotics}

Chlorhexidine mouthwash

50

\begin{tabular}{|l|c|c|}
\hline Complication & CHX & AMOX \\
\hline No complication & 38 & 42 \\
\hline Pain & 9 & 6 \\
\hline Swelling & 8 & 4 \\
\hline Trismus & 3 & 1 \\
\hline Alveolitis & 4 & 3 \\
\hline Infection & 4 & 3 \\
\hline Chlorhexidine =CHX Amoxicillin =AMOX &
\end{tabular}


Patients were prescribed $1 \mathrm{~g}$ of Paracetamol with codeine and 400mg of Ibuprofen as analgesics and postoperative instructions of care were given, which included rinsing with warm saline three times daily starting from the day after surgery.

A recall visit was scheduled seven days later and any postoperative complications were noted in a questionnaire. Pain, swelling, trismus, alveolitis and surgical site infection were recorded.

Trismus was defined as an inability to clear an inter-incisal distance of at least $2 \mathrm{~cm}$. Alveolar osteitis was defined as pain that arises 2-5 days after surgery, the presence of necrotic tissue and/or exposed bone, and the absence of clot.13,14 Infection was defined as a purulent discharge at the extraction site with/ without painful induration. ${ }^{13}$

If any complication arose before the scheduled recall visit, the patient was asked to report back to the hospital to receive appropriate treatment and postoperative antibiotics were then prescribed, if required. The complication was recorded on a data collection sheet.

Data collected were analyzed statistically using Stata13.1 for Windows. Participants were described using frequencies and percentage for categorical predictors, and means and standard deviations for continuous variables.

Associations were investigated using appropriate statistical tests such as the Student's t-test and Fisher's exact test for categorical predictors and ANOVA test for equal variance to analyze any significant differences inter-groups. Statistical tests used were two sided and $p$ values $\leq 0.01$ were considered significant.

\section{RESULTS}

The sample included almost equal numbers of males $(n=48,48 \%)$ and females $(n=52,52 \%)$ with ages ranging from 18-50 years (mean: $27.75+/-5.79$ ). Many patients presented with more than one complication. Alveolitis was recorded in the presence or absence of infection. For the purpose of this study, the main focus was on infection as a complication.

In the control group, to whom had been administered a preoperative rinse of chlorhexidine, four patients presented with postoperative infections whereas in the test group who had consumed a bolus of preoperative antibiotics, three patients presented with postoperative infections. The infection rate recorded was $8 \%$ for the chlorhexidine group and $6 \%$ for the antibiotic group. The Fisher's exact test between the two groups of patients demonstrated a $p$ value $>0.01$ indicating no significant difference in infection rates between the chlorhexidine group and the antibiotic group. In addition, there was no association between the presence of infection and the following variables: Gender $(p=1.00)$, Type of impaction $(p=0.187)$, Indications for extraction $(p=0.651)$, Time taken $(p=0.07)$, Medications used $(p=1.00)$.
The mean duration of the surgery was 22.1 (+/- 5.79) minutes. The minimum and maximum times to complete the procedure were recorded as 5 and 54 minutes respectively.

The most common indication for extraction was pericoronitis $(n=39,39 \%)$. Mesioangular impactions $(n=46,46 \%)$ followed by horizontal impactions $(n=37,37 \%)$ were the highest incidences of angulations recorded.

An independent Student-t test demonstrated a marginal association $(p=0.01)$ between the mean age of patients that presented for mandibular third molar extraction and their gender.

No association existed between the mean age and the different types of impaction was revealed by the Anova test of equal variance $(p=0.39)$.

Moreover, the Fisher's exact test showed no association between the type of impaction and the gender distribution of participants $(p=0.467)$ or the type of impaction $(p=0.07)$.

\begin{tabular}{|c|c|c|c|c|}
\hline \multirow{2}{*}{ Variable } & \multicolumn{2}{|c|}{ Presence of Infection } & \multirow{2}{*}{ Total } & \multirow{2}{*}{$p$-value } \\
\hline & Yes & No & & \\
\hline \multicolumn{5}{|l|}{ Sex } \\
\hline Female & $4(7.69 \%)$ & $48(92.31 \%)$ & 52 & \multirow{2}{*}{1.00} \\
\hline Male & $3(6.25 \%)$ & 45 (93.75\%) & 48 & \\
\hline Age (mean, std) & $26.85(5.21)$ & $27.82(5.85)$ & & 0.67 \\
\hline \multicolumn{5}{|l|}{ Type of Impaction } \\
\hline Vertical & 1 & 36 & 37 & \multirow{4}{*}{0.187} \\
\hline Horizontal & 1 & 13 & 14 & \\
\hline Mesioangular & 4 & 42 & 46 & \\
\hline Distoangular & 1 & 2 & 3 & \\
\hline \multicolumn{5}{|l|}{ Indication } \\
\hline Pericoronitis & 4 & 35 & 39 & \multirow{9}{*}{0.651} \\
\hline Abscess & 0 & 3 & 3 & \\
\hline Caries & 1 & 33 & 34 & \\
\hline Periodontal disease & 1 & 9 & 10 & \\
\hline Root resorption & 0 & 2 & 2 & \\
\hline Atypical facial pain & 1 & 5 & 6 & \\
\hline Oral pathology & 0 & 1 & 1 & \\
\hline Prophylactic removal & 0 & 4 & 4 & \\
\hline Tooth in line of fracture & 0 & 1 & 1 & \\
\hline $\begin{array}{l}\text { Time taken in minutes } \\
\text { (median, IQR) }\end{array}$ & $28(20-35)$ & $20(15-26)$ & & 0.07 \\
\hline \multicolumn{5}{|l|}{ Medication } \\
\hline Antibiotic & 3 & 47 & 50 & \multirow[t]{2}{*}{1.00} \\
\hline Chlorhexidine mouthrinse & 4 & 46 & 50 & \\
\hline
\end{tabular}




\section{DISCUSSION}

This study attempted to compare the efficacy in prophylaxis of two different therapeutic strategies prior to mandibular third molar extraction:

1. A one minute oral rinse with $15 \mathrm{ml}$ of chlorhexidine mouthwash

2. A single preoperative bolus of $2 g$ Amoxicillin

One hundred and ten patients were included in the study. Seven patients did not attend their recall visit and were excluded from the study. Three patients had an operating time for extraction of above one hour and were not included due to increased exposure to bacteria within the oral cavity which could compromise the results. In addition, the procedures were performed in the pre-surgical absence of pericoronitis or active infection. The infection rate recorded in this study showed no significant difference between the chlorhexidine group and the antibiotics group ( $p>0.01$ ).

Based on this finding, it can be deduced that the results in terms of efficacy obtained with either antimicrobial therapy is comparable and therefore an assessment of their mechanism of action, risk and cost benefits was considered before making a recommendation.

Chlorhexidine gluconate is a well-known antimicrobial agent with a broad spectrum of activity against both Gram positive and Gram negative bacteria, facultative anaerobes and aerobes, yeasts as well as certain viruses including HIV. ${ }^{15}$ It exists in the form of cations and readily binds to the negatively charged particles of the oral mucosa and bacteria exhibiting an immediate as well as a prolonged antimicrobial effect on a broad spectrum of bacteria and fungi. ${ }^{15}$ Its use in the routine disinfection of patients and medical devices in ICU or in surgical scrubs has been well established. ${ }^{16}$ In dentistry, chlorhexidine in the form of an oral rinse is often used for prophylaxis or in the treatment of oral infections.

In this study, a $0.2 \%$ oral rinse of chlorhexidine gluconate was used as a prophylaxis. At this concentration, chlorhexidine displays bactericidal properties by disrupting the function of cell membranes and altering the osmotic equilibrium of bacteria. ${ }^{15}$ The rationale behind its use in this study was to reduce the oral bacterial load at the surgical site, thereby decreasing bacteraemia which harbours significant risks for post extraction infections. ${ }^{17}$ Organisms that have shown high susceptibility to chlorhexidine include several species of staphylococci, streptococci and various anaerobes which are important constituents of the complex oral flora. ${ }^{18}$

Several studies have demonstrated that a preoperative rinse with $0.2 \%$ chlorhexidine has significant antimicrobial effects on the oral flora and post extraction bacteraemia. ${ }^{10,17}$ In 1997, the American Heart Association (AHA) recommended the use of an antiseptic mouthwash to reduce bacteraemia prior to any dental manipulation, before modifying the recommendations in 2007. In 2006, the British Society for Antimicrobial Chemotherapy (BSAC) recognized the importance of a preoperative rinse with a $0.2 \%$ chlorhexidine mouthwash before any dental procedures in patients at high risk of infective endocarditis.19 Conversely, it has also been suggested that forceful rinsing with a chlorhexidine mouthwash may actually produce bacteraemia but there is no substantial evidence to confirm this hypothesis. ${ }^{17}$
In addition to its broad antibacterial spectrum and substantivity, one of the major advantages of chlorhexidine is the absence of resistance to the microorganisms it affects. ${ }^{20}$ The few and rare documented adverse reactions to chlorhexidine include hypersensitivity, unpleasant taste or tooth discolouration..$^{15}$ Also, there is no evidence to contradict the use of chlorhexidine mouthwash in pregnant or lactating women. ${ }^{15}$

As opposed to the few studies published on the efficacy of chlorhexidine prophylaxis in third molar surgery, 10,14 there is a plethora of studies on antibiotic prophylaxis. ${ }^{3,4,6,21}$ However, its usefulness in preventing postoperative infections continues to remain a matter of debate. Different treatment protocols and different antibiotics have been used and conflicting information exists regarding its benefit. Although there are definite recommendations by the American Heart Association and American Dental Association/ American Academy of Orthopaedic Surgeons (ADA/ AAOS) for antibiotic prophylaxis in the prevention of infective endocarditis and prosthetic joint infection, the guidelines for prophylactic antibiotics to prevent surgical site infection in oral and maxillofacial surgery are less clear. ${ }^{22}$ It is widely accepted that surgical site infections are caused by the patients' own endogenous flora and the purpose of prophylaxis is to considerably reduce the systemic level of the infective pathogens in the tissues at the operative site..$^{23,24}$

The choice of antibiotic is greatly influenced by the bacteria present at the operative site. Peterson ${ }^{1}$ has set forth certain criteria when choosing an antibiotic for prophylaxis. He advocates for the correct antibiotic with the narrowest antibacterial spectrum. In addition, a high enough dosage must be administered at the most appropriate time and with the shortest exposure. Amoxicillin has been the gold standard for treatment of infections or prophylaxis in dentistry due to its high efficacy against Gram-positive streptococcus and staphylococcus species as well as several Gram-negative bacteria which are common isolates in oral infection. ${ }^{25}$ In addition, its good absorption in the gastrointestinal tract coupled with its capacity in reaching fast and effective concentrations at the site it targets has made it an antibiotic of choice. ${ }^{26}$

A $2 \mathrm{~g}$ amoxicillin regimen one hour before surgery was used in this study based on the AHA/ADA/AAOS guidelines. ${ }^{11}$ Similar protocols were used in several studies with varying results on the efficacy of the prophylactic treatment. ${ }^{8,27}$ Ren et $a^{28}$ performed a meta-analysis of 23 studies on the effectiveness of antibiotic prophylaxis in third molar surgery and concluded that when systemic antibiotics are administered before surgery, they are effective in reducing the frequency of wound infections. In contrast, in a review of published clinical trials on the efficacy of antibiotic prophylaxis, Oomens et a/29 reported a lack of evidence to support the use of prophylactic antibiotics in lower third molar surgery.

The use of amoxicillin in the prevention of infection in dentistry is a source of major debate due to the development of antibacterial resistance and other adverse reactions such as anaphylaxis or toxicity. ${ }^{2}$ The World Health Organisation's (WHO) 2014 global report on antibiotic resistance highlighted resistance as a major global threat to worldwide public health with new resistance mechanisms continuously emerging and spreading globally at an alarming rate. ${ }^{30}$ The development of resistance over 
the years is most probably due to overuse or misuse of antibiotics. ${ }^{24}$ There is no doubt that the poor antibiotic prescribing practices by dentists, often motivated by factors ranging from inadequate knowledge to social factors, contribute significantly towards the formation of multiresistant bacterial strains. ${ }^{31}$

Judicious use of antibiotic prophylaxis based on evidence rather than dogma is imperative. The published infection rate associated with third molar surgery ranges between 1\%-12.6\%. ${ }^{2}$ The infection rate for amoxicillin and chlorhexidine prophylaxis in this study was $6 \%$ and $8 \%$ respectively and falls within the expected rate of infection for third molar removals. Hence the efficacy of both regimens is comparable.

In view of the potential harmful complications associated with the use of antibiotics for prophylaxis and the relatively low rate of infection posed by third molar surgery, the use of amoxicillin is not warranted for such procedures. However, chlorhexidine gluconate which is a cheap, safe and broad spectrum antiseptic is recommended to reduce the oral bacterial count at the surgical site prior to making an incision. ${ }^{32}$

Moreover, a session of professional scaling is desirable before third molar surgery to decrease the oral bacterial load. Ultimately, chlorhexidine must be an adjunct to proper surgical technique in an aseptic environment.

As far as demographics are concerned, the ratio of male to female in this study was 1:1.08, which shows an almost equal distribution between the two gender groups. This is in agreement with most studies that have reported no sexual predilection for impacted third molars. ${ }^{33}$ Other studies have shown a higher predilection for females and it is believed that mandibular growth for females stops by the time third molars start erupting, resulting in a lack of space for the tooth to erupt. ${ }^{33-35}$

The most common type of impaction recorded was the mesioangular type (46\%) followed by the vertical type (37\%). Mesioangular impactions are most probably caused by the late development and maturation of the tooth germ with a resulting lack of space for the tooth to erupt in a normal position. ${ }^{33}$ The results are similar to those found by Hashemipour et $a^{/ 33}$ and Gbotolorun et $a l^{36}$ in Iran and Nigeria respectively. This is in contrast to other studies that demonstrated the vertical type of impaction to be more common. ${ }^{34,37}$ Differences in methods of classification for angulation used, variation in genetics between the populations studied or the type of food consumed in the different geographical areas may account for the discrepancies. ${ }^{33}$ This study also demonstrated that no correlation exists between the type of impaction and the different variables of age and gender.

The mean age of the subjects was 27.75 with a standard deviation of 5.79. The age group 25-30 years had the highest proportion of patients with impacted teeth. The delayed manifestation of patients for impacted teeth removal may be due to a lack of Oral Health Education. In certain cases there is also the wrong perception that the tooth is still growing and will erupt eventually. Moreover, due to apprehension for dental treatment, most patients in Mpumalanga visit the dentist only when they are inflicted with pain.
Pericoronitis followed by caries was the most frequent indication of the need for extractions. These results are comparable to those found by Krishnan et $a^{38}$ where pericoronitis was the most frequent reason for extraction followed by caries. The high prevalence of these pathologies is not uncommon since they usually present with symptoms of pain which force patients to seek dental assistance. Pericoronitis in the patients seen is probably exacerbated by the accumulation of bacteria and food under the operculum. Similarly caries may have formed due to poor oral hygiene resulting in a build-up of food and debris that become impacted around the partially erupted tooth.

The implementation of oral health education in both personal and community based programs remains key to informing patients on the importance of oral hygiene. A paradigm shift from interventional dentistry towards preventive oral health within the hospital setting will undoubtedly assist in curtailing the burden of oral diseases in the community who should enjoy ready access to oral health care.

\section{CONCLUSION}

This study demonstrated that the prophylactic use of a chlorhexidine mouthrinse and amoxicillin in third molar surgery are equally effective in keeping postoperative infections to a minimum in medically competent patients. Indeed, a single dose of antibiotic prophylaxis failed to show any additional clinical advantage compared with the use of a chlorhexidine mouthrinse in reducing infections. Routine administration of prophylactic amoxicillin therapy in non-immunocompromised patients is therefore not recommended as the risks associated outweigh the benefits. A session of preoperative professional cleaning and a routine rinse with $0.2 \%$ chlorhexidine mouthrinse appears slightly more beneficial than antibiotic administration.

\section{References}

1. Ness GM, Peterson LJ. Impacted teeth In: Miloro M, Ghali GE Larsen PE, Waite PD, editors. Peterson's Principles of Oral and Maxillofacial Surgery. 2nd edition. Ontario: BC Decker: 2004: Chapter 8: 139-56.

2. Martin MV, Kanatas AN, Hardy P. Antibiotic prophylaxis and third molar surgery. British Dental J. 2005; 198: 327-30.

3. Monaco G, Tavernese L, Agostini R, Marchetti C. Evaluation of antibiotic prophylaxis in reducing postoperative infection after mandibular third molar extraction in young patients. J Oral Maxillofac Surg. 2009; 67: 1467-72.

4. Lopez-Cedrun JL, Pijoan JI, Fernandez S, Santamaria J, Hernandez G. Efficacy of Amoxicillin treatment in preventing postoperative complications in patients undergoing third molar surgery: A prospective, randomized, double blind controlled study. J Oral and Maxillofac Surg. 2011; 69: e5-14.

5. Siddiqui A, Morkel JA, Zafar S. Antibiotic prophylaxis in third molar surgery: A randomised double blind placebo-controlled clinical trial using split-mouth technique. Int J Oral Maxillofac Surg. 2010; 39: 107-14.

6. Pasupathy S, Alexander M. Antibiotic prophylaxis in third molar surgery. J Craniofac Surg. 2011; 22: 551-3.

7. Arteaogoitia L, Ramos E, Santamaria G, Barbier L, Alvarez J, Santamaria J. Amoxicillin/clavulanic acid 2000/125 mg to prevent complications due to infection following completely bone-impacted lower third molar removal: a clinical trial. Oral Surg Oral Med Oral Pathol Oral Radiol J. 2015; 119: 8-16.

8. Ataoqlu H, Oz YG, Candirli C, Kiziloqlu D. Routine antibiotic prophylaxis is not necessary during operations to remove third molars. Br J Oral Maxillofac surg. 2008; 46: 133-5.

9. Olusanya AA, Aritiba JT, Fasola OA, Akadiri AO. Prophylaxis versus pre-emptive antibiotics in third molar surgery: a randomised control study. Niger Postgrad Med J. 2011; 18: 105-10. 
10. Tuna A, Delibasi C, Arslan A, Gurol Y, Tazequn Tekkanat Z. Do antibacterial mouthrinses affect bacteraemia in third molar surgery? A pilot study. Aust Dental J. 2012; 57: 435-9.

11. Duvall NB, Fisher TD, Hensley D, Hancock RH, Vandewalle KS. The comparative efficacy of $0.12 \%$ Chlorhexidine and Amoxicillin to replace the incidence and magnitude of bacteraemia during third molar extractions: A prospective, blind randomised clinical trial. Oral Surg Oral Med Oral Pathol Oral Radiol. 2013; 115: 752-63.

12. Maharaj B, Coovadia $Y$, Vayej AC. A comparative study of amoxicillin, clindamycin and chlorhexidine in the prevention of post extraction bacteraemia. J Cardiovasc Afr. 2012; 23: 491-4.

13. Blondeau F, Daniel N. Extraction of impacted mandibular third molars: Postoperative complications and their risk factors. J of Can Dent Assoc. 2007; 73: 325a-e.

14. Metin M, Sener IJ. Comparison of two chlorhexidine rinse protocols on the incidence of alveolar osteitis following surgical removal of impacted third molars. J Contemp Dent Prac. 2006; 7: 79-86.

15. Yildirim A, Metzler P, Lubbers H, Yildirim V. Digluconate de Chlorhexidine- histoire, Mecanisme d'action et risques. Swiss Dental J. 2015; 125: 830-1.

16. Karki S, Cheng AC. Impact of non rinse skin cleansing with chlorhexidine gluconate on prevention of health care associated infections with multi resistant organisms: a systematic review. Hospital Infection J. 2012; 82: 71-84.

17. Tomas I, Alvarez M, Limeres J. Effect of a Chlorhexidine mouthwash on the risk of post extraction bacteremia. Infect Control Hosp Epidemiol J. 2007; 28: 577-82.

18. Mc Bain AJ, Bartolo RG, Gilbert P. Effects of a Chlorhexidine Gluconate mouthwash on the vitality and antimicrobial susceptibility of in vitro oral bacterial eco systems. Appl Environ Microbiol J. 2003; 69: 4770-6.

19. Barbosa M, Prada-Lopez I, Alvarez M., Amaral B, Maria de Los Angeles C, Tomas I. Post-tooth extraction bacteraemia: A randomized clinical trial on the efficacy of chlorhexidine prophylaxis. A Peer-Reviewed, Open Access J. 2012; 10: e0124249.

20. Watanabe E, Nascimento AP, Guerreiro-Tanomaru JM, Razaboni AM, De Andrade D, Tanomaru-Filho M. Antiseptic mouthwashes: in vitro antibacterial activity. Acta odontol Latinoam J. 2015; 18: 180-4.

21. Lawler B, Sambrook PJ, Goss AN. Antibiotic prophylaxis for dento alveolar surgery: Is it indicated? Aust Dental J. 2005; 50: S54-9.

22. Smith JA. Antibiotic prophylaxis to prevent surgical site infection in Maxillofacial and Oral Surgery. In: Bagheri S, Bryan Bell $\mathrm{R}$, Khan $\mathrm{H}$, editors. Current therapy in Oral and Maxillofacial Surgery. St Louis: Elsevier Saunders: 2011: Chapter 8: 67-78.

23. Mohammed A, Dusara K. Can antibiotic prophylaxis prior to surgical removal of teeth reduce the incidence of postoperative infections? J of Dentistry, Oral disorders and Therapy. 2014; $1: 5$.

24. Carlson MA. Prophylactic antibiotics in surgery. Annual Review of Medicine J. 1993; 44: 385-93.

25. Ramu C, Padmanabhan TV. Indications of antibiotic prophylaxis in dental practice- Review. Asian Pac J Trop Biomed. 2012; 2: 749-54.

26. Sweeney L, Dave J, Chambers PA, Heritage J. Antibiotic resistance in general dental practice - A cause for concern? Chemotherapy J. 2004; 53: 563-76.

27. Bortoluzzi MC, Capella DL, Barbieri T, Pagliarini M, Cavalieri T, Manfro R. A single dose of Amoxicillin and Dexamethasone for prevention of postoperative complications in third molar surgery: A randomized, double blind, placebo controlled clinical trial. J Clin Med Res. 2013; 5: 26-33.

28. Ren YF, Malmstrom HS. Effectiveness of antibiotic prophylaxis in third molar surgery: A Meta-Analysis of randomized clinical trials. J Oral Maxillofac Surg. 2007; 65: 1909-21.

29. Oomens MA, Forouzanfar T. Antibiotic prophylaxis in third molar surgery: a review, Oral Surg, Oral Med, Oral Pathol and Oral Radiol J. 2012; 114: e5-12.
30. World Health Organisation. Antimicrobial Resistance. Fact sheet no 194. http:/www.who.int/mediacentre/factsheets/ fs194/en/ [Accessed on: 29th May 2014].

31. Dar-Odeh NS, Abu Hammad OA, Al-Omiri MK, Kharaisat AS, Shehabi AA. Antibiotic prescribing practices by dentists: a review. The Risk Management J. 2010; 6: 301-6.

32. Gusberti FA, Sampathkumar P, Siegrist BE, Lang NP. Microbiological and clinical effects of chlorhexidine digluconate and hydrogen peroxide mouthrinses of developing plaque and gingivitis. Clin Periodontol J. 1988; 15: 60-7.

33. Hashemipour MA, Tahmasbi-Arashlow M, Fahimi-Hanzaei F. Incidence of impacted mandibular and maxillary third molars: A radiographic study in a South East Iran population. Med Oral Patol Oral Cir Bucal. 2013; 13: 40-5.

34. Qirreish EJ. Radiographic Profile of symptomatic impacted mandibular third molars in the Western Cape, South Africa. Masters Degree Dissertation. Western Cape: University of Western Cape; 2005.

35. Quek SL, Tay CK, Tay CH, Toh SL, Lim KC. Pattern of third molar impaction in a Singapore Chinese population: A retrospective radiographic survey. Int J Oral Maxillofac Surg. 2003; 32: 548-52.

36. Gbotolorun OM, Olojede AC, Arotiba GT, Ladeinde $\mathrm{AL}$, Akinwande JA, Bangbose BO. Impacted 3rd molars: Presentation and postoperative complication at the Lagos University teaching hospital. Nig Q J Hosp Med. 2007; 17: 26-9.

37. Almendros-Marqes N, Berini- Aytes L, Gay-Escoda C. Evaluation of intraexaminer and interexaminer agreement on classifying lower third molars according to the systems of Pell and Gregory and of Winter. J Oral and Maxillofac Surg. 2008; 66: 893-9

38. Krishnan B, El Sheikh MH, El Gehani R, Orafi H. Indications for removal of impacted mandibular molars: a single institutional experience in Lybia. J Oral Maxillofac Surg. 2009; 8: 246-8. 